S. Williamson

Nagoya Math. J.

Vol. 46 (1972), 97-109

\title{
RAMIFIGATION THEORY FOR EXTENSIONS OF DEGREE p. II
}

\author{
SUSAN WILLIAMSON
}

Introduction. Let $k$ denote the quotient field of a complete discrete rank one valuation ring $R$ of unequal characteristic and let $p$ denote the characteristic of $\bar{R}$; assume that $R$ contains a primitive $p^{t h}$ root of unity, so that the absolute ramification index $e$ of $R$ is a multiple of $p-1$, and each Gallois extension $K \supset k$ of degree $p$ may be obtained by the adjunction of a $p^{t h}$ root.

The purpose of this paper is to assign to each Galois extension $K \supset k$ of degree $p$ an integer $f$ with $-1 \leq f \leq e p / p-1$ from which the ramification-theoretic properties of $K \supset k$ can be determined. Specifically, $f$ shall determine the unramified, wildly ramified, or fiercely ramified character of $K \supset k$, (see Thm. 1.11); moreover, the ramification number $i$ of $K \supset k$ shall have an expression in terms of $f$, (see Prop. 2.1).

Let $U^{(i)}$ for $i \geq 0$ denote the usual filtration on the units of $R$, and let $U^{(-1)}$ denote the set of prime elements of $R$. In a recent publication, ([5]), the author has studied the ramification-theoretic properties of a Galois extension $K \supset k$ of degree $p$ by constructing the integral closure $S$ of $R$ in $K$ from a judiciously chosen element $b$ of $U^{(x)}(-1 \leq x \leq p)$ whose $p^{t h}$ root defines $K$. The method for computing $S$ in [5] entails the construction of a chain of $g+1$ ring extensions of $R$ in $S$; the number $g$ is unique for $K \supset$ $k$, satisfies the inequality $0 \leq g \leq(e / p-1)-1$, and is called the conductor number of $K \supset k$.

The present paper makes use of the fact that $R$ is an Eisenstein extension of an unramified complete discrete rank one valuation ring in order to determine an alternate method for the construction of the integral closure $S$. In Section 1 we associate to each Galois extension $K \supset k$ of degree $p$ an integer $f$ with $-1 \leq f \leq e p / p-1$ called its absolute field exponent such that

Received June 2, 1971. 
$K$ may be obtained from $k$ by the adjunction of a judiciously chosen element $b$ of $U^{(f)}$. By computing $S$ from such an element $b$ of $R$, we prove the following main result.

TheOREm. Let $f$ denote the absolute field exponent of a Galois extension $K \supset k$ of degree $p$. Then

i) $K \supset k$ is wild if and only if $f$ is relatively prime to $p$

ii) $K \supset k$ is fierce if and only if $p$ divides $f$ and $f<e p / p-1$

iii) $K \supset k$ is unramified if and only if $f=e p / p-1$.

In Section 2 we relate the results of the present paper to those of [5]. More specifically, we relate the notions of absolute field exponent and conductor number by computing an expression for the ramification number of $K \supset k$ in terms of the absolute field exponent $f$, and then applying the results of Section 3 of [5].

The author's recent paper and her present paper provide a choice of two significantly different methods for computing the integral closures $S$ of $R$ in a Galois extension $K \supset k$ of degree $p$. Available information concerning an element $b$ whose $p^{t h}$ root defines the extension determines the proper choice of method.

The following notation shall be used throughout the paper. The multiplicative group of units of a ring $R$ shall be denoted by $U(R)$; the intermediate ring obtained by adjoining to $R$ an element $t$ of an overring of $R$ shall be denoted by $R[t]$; and, the residue class field of a local ring $R$ shall be denoted by $\bar{R}$.

Unless otherwise stated, $R$ shall always denote a complete discrete rank one valuation ring of unequal characteristic which contains a primitive $p^{t h}$ root of unity where $p$ denotes the characteristic of $\bar{R}$, and $S$ shall denote the integral closure of $R$ in a Galois extension $K$ of degree $p$ over the quotient field $k$ of $R$; $\Pi$ shall denote a prime element of $R$, $\Pi$ a prime element of $S$, and $e$ the absolute ramification index of $R$. The usual filtration on $U(R)$ shall be denoted by $U^{(i)}(i \geq 0)$ and $U^{(-1)}$ shall denote the set of prime elements of $R$.

In [5], the author has defined the quotient field extension of an extension of discrete rank one valuation rings to be fiercely ramified if the residue class field extension has a non-trivial inseparable part. For further details, the reader may refer to [5]. 
1. The absolute field exponent. Throughout this section $K \supset k$ shall always denote a $p^{t h}$ root extension, where $k$ is the quotient field of a complete discrete rank one valuation ring $R$ of unequal characteristic containing a primitive $p^{t h}$ root of unity and $p$ is the characteristic of $\bar{R}$, and $S$ shall always denote the integral closure of $R$ in $K$. The purpose of this section is to define for each Galois extension $K \supset k$ of degree $p$ an integer $f$ with $-1 \leq f \leq e p / p-1$ called its absolute field exponent, and to establish a criterion for determining if $K \supset k$ is unramified, wild, or fierce in terms of its absolute field exponent.

In [5], the author has assigned to each such extension $K \supset k$ an integer $x$ with $-1 \leq x \leq p$ called its field exponent; the notions of field exponent and absolute field exponent coincide in the case when $k$ has absolute ramification index $p-1$. We shall make use of results established in [5] in our study of the absolute field exponent.

The first three lemmas concern elements $b$ whose $p^{\text {th }}$ roots define the extension $K \supset k$. Lem. 1.1 follows at once from Prop. 1.3 of [5].

Lemma 1.1. If $K \supset k$ is a Galois extension of degree $p$, then $K=k\left(b^{1 / p}\right)$ for some element $b$ in $U^{(-1)}$ or in $U^{(0)}$.

Lemma 1.2. i) If $b$ is in $U^{(-1)}$, then $k\left(b^{1 / p}\right) \supset k$ is wild of degree $p$, and $k\left(b^{1 / p}\right) \neq k\left(b_{1}{ }^{1 / p}\right)$ for every element $b_{1}$ of $U^{(0)}$.

ii) If $b$ is in $U^{(0)}, b_{1}$ is in $U^{(1)}$, and $k\left(b^{1 / p}\right)=k\left(b_{1}{ }^{1 / p}\right)$, then $\bar{b}$ has a $p^{\text {th }}$ root in $\bar{R}$.

iii) If $b$ is in $U^{(0)}$ and $X^{p}-\bar{b}$ is irreducible over $\bar{R}$, then $k\left(b^{1 / p}\right) \supset k$ is fierce of degree $p$.

Proof. If $b$ is in $U^{(-1)}$ then $b^{1 / p}$ is a root of an Eisenstein polynomial of degree $p$, from which it follows that $K \supset k$ is wild of degree $p$. If $b$ is in $U^{(0)}$ and $X^{p}-\bar{b}$ is irreducible over $\bar{R}$, then $\bar{S} \supset \bar{R}$ is purely inseparable of degree $p$. The remaining assertions are restatements of parts ii) and iii) of Lem. 1.5 of [5].

Remark 1.3. If $b$ is in $U^{(-1)}$, then the integral closure $S$ of $R$ in $k\left(b^{1 / p}\right)$ is $S=R\left[b^{1 / p}\right]$. If $b$ is in $U^{(0)}$ and $X^{p}-\bar{b}$ is irreducible over $\bar{R}$, then the integral closure $S$ of $R$ in $k\left(b^{1 / p}\right)$ is $S=R\left[b^{1 / p}\right]$.

The above expressions for the integral closure have been established in 
Prop. 2. $6 A$ of [5]; we shall make use of them in our study of the ramification number of $K \supset k$ in Section 2.

Lemma 1.6 pertains to extensions $K \supset k$ of degree $p$ obtained by the adjunction of a $p^{t h}$ root of an element $b$ of $R$ present in $U^{(1)}$. Recall that the complete discrete rank one valuation ring $R$ is an. Eisenstein extension of an unramified complete discrete rank one valuation ring $R_{0}$ (see Thm. 31.12 p. 111 of [3]). Let $e$ denote the ramification index of the totally ramified extension $R \supset R_{0}$; then $R=R_{0}[\pi]$ where $\pi$ denotes a prime element of $R,\left\{1, \pi, \cdots, \pi^{e-1}\right\}$ is a free basis for $R$ over $R_{0}, \pi^{e} R=p R$, and $\bar{R}=\bar{R}_{0}$, (see Thm. 1 p. 23 of [2]). Moreover, $e$ is the absolute ramification index of $R$.

Facts 1.4 and 1.5 shall be used in the proof of Lem. 1.6.

FACT 1.4. If $b_{1}$ and $b_{2}$ are elements of $U(R)$ such that $b_{1} \equiv b_{2} \bmod \pi^{(e p / p-1)+1} R$, then $k\left(b_{1}{ }^{1 / p}\right)=k\left(b_{2}{ }^{1 / p}\right)$.

Proof. Since $b_{1}$ and $b_{2}$ are in $U(R)$, the congruence $b_{1} \equiv b_{2} \bmod \pi^{(e p / p-1)+1} R$ implies that $b_{1} / b_{2} \equiv 1 \bmod \pi^{(e p / p-1)+1} R$, from which it follows that $b_{1} / b_{2}$ has a $p^{t h}$ root in $R$ according to Lem. 1.2 of [5]. The fact that $b_{1}$ and $b_{2}$ differ multiplicatively by a $p^{t h}$ power from $k$ implies that $k\left(b_{1}{ }^{1 / p}\right)=k\left(b_{2}{ }^{1 / p}\right)$.

FACT 1.5. If $b$ is an element of $R$, then there exists an element $c$ of $R$ of the form $c=\sum y_{i} \pi^{i}(0 \leq i \leq e p / p-1)$ with each $y_{i}$ in $U\left(R_{0}\right) \cup\{0\}$ which satisfies the congruence $c \equiv b \bmod \pi^{(e p / p-1)+1} R$. If $b$ is in $U^{(1)}$, then $c$ may be chosen so that $y_{0}=1$.

Proof. Since $\left\{1, \pi, \cdots, \pi^{e-1}\right\}$ is an $R_{0}$-module basis for $R$, the element $b$ may be written in the form $b=\sum b_{i} \pi^{i}(0 \leq i \leq e-1)$ with each $b_{i}$ in $R_{0}$. If each $b_{i}$ is in $U\left(R_{0}\right) \cup\{0\}$, then $c=b$ satisfies the assertion. Otherwise, we may consider the least positive integer $h$ such that $b_{h}$ is not in $U\left(R_{0}\right) \cup$ $\{0\}$. Since $b_{h}$ is in $p R_{0}$, the element $b$ satisfies the congruence $b \equiv \sum b_{i} \pi^{i}$ $\bmod \pi^{h+1} R(0 \leq i \leq h-1)$; we may consider therefore an element $\tilde{b}$ of $R$ of the form $\tilde{b}=\sum \tilde{b}_{i} \pi^{i}(0 \leq i \leq e p / p-1)$ with each $\tilde{b}_{i}$ in $R_{0}, \tilde{b}_{i}=b_{i}$ for $0 \leq i$ $\leq h-1$, and $\tilde{b}_{h}=0$, which satisfies the congruence $\tilde{b} \equiv b \bmod \pi^{(e p / p-1)+1} R$. If each $\tilde{b}_{i}$ is in $U\left(R_{0}\right) \cup\{0\}$ for $0 \leq i \leq e p / p-1$, then $c=\tilde{b}$ satisfies the assertion. Otherwise, we may consider the least positive integer $m$ such that $\tilde{b}_{m}$ is not in $U\left(R_{0}\right) \cup\{0\}$. Observe that $h<m$, so that by proceeding in this way we may obtain, after finitely many steps, an element which $c$ satisfies the statement of our assertion. 
If $b$ is in $U^{(1)}$, then $b=1+r \pi$ for some element $r$ of $R$. By applying the first part of this fact to $r$, we may produce an element $c$ of the desired form which satisfies the congruence $c \equiv b \bmod \pi^{(e p / p-1)+1} R$.

Lemma 1.6. Assume the notation introduced above. Let $K \supset k$ be an extension of degree $p$ defined by $K=k\left(b^{1 / p}\right)$ for some element $b$ of $U^{(1)}$. Then $K=k\left(b_{1}^{1 / p}\right)$ for some element $b_{1}$ of $U^{(1)}$ of the form $b_{1}=1+\sum x_{i} \pi^{i}(1 \leq i \leq e p / p-1)$ where the $x_{i}$ are elements of $R_{0}$ such that

i) each $x_{i}$ is in $U\left(R_{0}\right) \cup\{0\}$, and the $x_{i}$ are not all zero

ii) $X^{p}-\bar{x}_{i}$ is irreducible over $\bar{R}_{0}$ for each $i$ divisible by $p$ such that $i<e p /$ $p-1$ and $x_{i} \neq 0$.

Proof. By combining Facts 1.4 and 1.5 we may consider an element $c$ of $R$ of the form $c=1+\sum y_{i} \pi^{i}(1 \leq i \leq e p / p-1)$ with the $y_{i}$ in $U\left(R_{0}\right) \cup\{0\}$ such that $k\left(c^{1 / p}\right)=k\left(b^{1 / p}\right)$. Observe that the $y_{i}$ are not all zero; for if $y_{i}=$ 0 for each $i$, then $k\left(c^{1 / p}\right)=k$, which contradicts the assumption that $K \supset k$ has degree $p$. If $X^{p}-\bar{y}_{i}$ is irreducible over $\bar{R}_{0}$ for every $i$ divisible by $p$ and less than $e p / p-1$ for which $y_{i} \neq 0$, then $b_{1}=c$ satisfies the assertion of this lemma.

Otherwise, we may consider the least positive integer $h$ divisible by $p$, less than $e p / p-1$, for which $y_{h} \neq 0$ and $X^{p}-\bar{y}_{h}$ is reducible over $\bar{R}_{0}$. We proceed to show that $c$ can be replaced by an element $c_{1}$ of $U^{(1)}$ of the form $c_{1}=\Sigma \gamma_{i} \pi^{i}(1 \leq i \leq e p / p-1)$, where the $\gamma_{i}$ are in $U\left(R_{0}\right) \cup\{0\}$ and are not all zero, such that for every $i \leq h$ divisible by $p$ for which $\gamma_{i} \neq 0$, the polynomial $X^{p}-\bar{\gamma}_{i}$ is irreducible over $\bar{R}_{0}$. Since $X^{p}-\bar{y}_{h}$ is reducible over $\bar{R}_{0}$, we may consider an element $y$ of $R_{0}$ such that $\bar{y}^{p}=\bar{y}_{h}$, i.e. such that $y^{p} \equiv$ $y_{h} \bmod p R$. Define the element $\tilde{c}$ of $R$ by $\tilde{c}=c\left(1-y \pi^{h / p}\right)^{p}$, and observe that $k\left(\tilde{c}^{1 / p}\right) \equiv k\left(c^{1 / p}\right)$ because $\tilde{c}$ and $c$ differ multiplicatively by a $p^{t h}$ power from $k$. By expanding $\left(1-y \pi^{h / p}\right)^{p}$ according to the binomial theorem, we obtain the congruence $\tilde{c} \equiv c\left(1-y^{p} \pi^{h}\right) \bmod \pi^{e+(h / p)} R$ since $p R=\pi^{e} R$. It is easy to verify that $h+1 \leq e+h / p$ if and only if $h<e p / p-1$. Therefore the fact that $h<e p / p-1$ now implies that $\tilde{c} \equiv c-y^{p} \pi^{h} \bmod \pi^{h+1} R$, because $c$ is in $U^{(1)}$. Since $c=1+\sum y_{i} \pi^{i}(1 \leq i \leq e p / p-1)$ and $y^{p}=y_{h} \bmod p R$, it now follows that $\tilde{c} \equiv 1+\sum y_{i} \pi^{i}+\left(y_{h}-y^{p}\right) \pi^{h} \bmod \pi^{h+1} R \quad(1 \leq i \leq h-1) \equiv 1$ $+\sum y_{i} \pi^{i} \bmod \pi^{h+1} R(1 \leq i \leq h-1)$. Now we may define he desired element $c_{1}$. According to the preceeding congruences we may write $\tilde{c}$ in the form $\tilde{c}=1+\sum \gamma_{1} \pi^{i}+r \pi^{h+1}(1 \leq i \leq h)$ for some element $r$ of $R$, where $\gamma_{i}=y_{i}$ 
$(1 \leq i \leq h-1)$ and $\gamma_{h}=0$. An application of Fact 1.5 yields the existence of an element of the form $\sum \gamma_{i} \pi^{i}(h+1 \leq i \leq e p / p-1)$ with the $\gamma_{i}$ in $U\left(R_{0}\right)$ $\cup\{0\}$ which satisfies the congruence $\sum_{i=h+1}^{e p / p-1} \gamma_{i} \pi^{i} \equiv r \pi^{h+1} \bmod \pi^{(e p / p-1)+1} R$. Define the element $c_{1}$ of $U^{(1)}$ by $c_{1}=1+\sum \gamma_{i} \pi^{i}(1 \leq i \leq e p / p-1)$. Observe that $c_{1}=\tilde{c} \bmod \pi^{(e p / p-1)+1} R$, so that $K=k\left(c_{1}^{1 / p}\right)$ according to Fact 1.4 because $K=k\left(\tilde{c}^{1 / p}\right)$. Since $\gamma_{h}=0$ and $\gamma_{i}=y_{i}$ for $1 \leq i \leq h-1$, it is true that the polynomial $X^{p}-\bar{\gamma}_{i}$ is irreducible over $\bar{R}_{0}$ for each $i(1 \leq i \leq h)$ divisible by $p$ such that $\gamma_{i} \neq 0$. An argument similar to the one at the beginning of the proof shows that the elements $\gamma_{i}(1 \leq i \leq e p / p-1)$ are not all zero. If $X^{p}-$ $\bar{\gamma}_{i}$ is irreducible over $\bar{R}_{0}$ for every $i<e p / p-1$ which is divisible by $p$ and for which $\gamma_{i} \neq 0$, then $b_{1}=c_{1}$ satisfies the assertion of this lemma.

Otherwise, we may consider the least positive integer $m$ less than $e p /$ $p-1$ and divisible by $p$ such that $\gamma_{m} \neq 0$ and $X^{p}-\bar{\gamma}_{m}$ is reducible over $\bar{R}_{0}$; observe that $h<m$. By means of the same technique used above to produce $c_{1}$ from $c$, we may produce an element $c_{2}$ of $U^{(1)}$ of the form $c_{2}=1+$ $\sum \delta_{i} \pi^{i}(1 \leq i \leq e p / p-1)$ such that $k\left(c_{2}{ }^{1 / p}\right)=k\left(c_{1}{ }^{1 / p}\right)$, where the $\delta_{i}$ are in $U\left(R_{0}\right)$ $\cup\{0\}$ and are not all zero, and the polynomials $X^{p}-\bar{\delta}_{i}$ are irreducible over $\bar{R}_{0}$ for every $i \leq m$ divisible by $p$ for which $\delta_{i} \neq 0$.

It follows from the inequality $h<m$, that by proceeding in this way we may finally obtain an element $b_{1}$ of $U^{(1)}$ which satisfies the assertion of this lemma.

Definition. An element $b$ of $U^{(1)}$ of the form $b=1+\sum x_{i} \pi^{i}(1 \leq i \leq$ $e p / p-1)$ with the $x_{i}$ in $R_{0}$ is said to be in normal form if the $x_{i}$ satisfy statements i) and ii) of Lem. 1.6.

The usefulness of Lem. 1.6 for the definition of the absolute field exponent motivates its name.

The following proposition concerning elements of $U^{(1)}$ shall be used to establish the main result (Thm. 1.11); its corollary (Lem. 1.9) shall be used to establish the uniqueness of the absolute field exponent in Prop. 1.10.

Proposition 1.7. Let $b=1+\sum x_{i} \pi^{i}(1 \leq i \leq e p / p-1)$ denote an element of $U^{(1)}$ in normal form, and let $f$ denote the least integer for which $x_{f} \neq 0$; let $K=$ $k\left(b^{1 / p}\right)$. Then

i) $K \supset k$ is wild of degree $p$ if and only if $f$ is relatively prime to $p$

ii) $K \supset k$ is fierce of degree $p$ if and only if $p$ divides $f$ and $f<e p / p-1$

iii) $K \supset k$ is unramified if and only if $f=e p / p-1$. Moreover, $K \supset k$ is 
unramified of degree $p$ if and only if $f=e p / p-1$ and the polynomial $X^{p}+\bar{v} X-$ $\bar{x}_{f}$ is irreducible over $\bar{R}_{0}$, where $v$ is the element of $U(R)$ defined by $v \pi^{e}=p$.

Proof. Recall (see Prop. 1.1 of [5]) that a $p^{t h}$ root $\beta$ of an element $b$ of $U^{(1)}$ satisfies an equality of the form $(\beta-1)^{p}=(b-1)+u v \pi^{e}(\beta-1)$ where $u$ is an element of the $R$-module $R\left(1, \beta, \cdots, \beta^{p-2}\right)$ which satisfies the congruence $u \equiv-1 \bmod (p, \beta-1) R[\beta]$, and $v$ is the element of $U(R)$ defined by $v \pi^{e}$ $=p$. The proceeding equality shall be used for establishing the asserted relationships between $f$ and the ramification-theoretic character of $K \supset k$.

First we shall prove that if $f$ is relatively prime to $p$, then $K \supset k$ is wild of degree $p$ by constructing a prime element $\Pi$ of the integral closure $S$ of $R$ in $K$. By applying the division algorithm to $f$ and $p$ we may obtain (unique) integers $q$ and $t$ such that $f=q p+t$ where $0 \leq t<p$. Observe that $q \geq 0$ because $f \geq 1$, and that $0<t$ because $(f, p)=1$. The element $\theta$ of $K$ defined by $\theta=(\beta-1) \pi^{q}$ shall be useful for constructing $\Pi$. We proceed to show that $\theta$ is a non-unit of $S$ and that $\theta^{p}$ is in $\pi^{t} U(S)$. Consider the element $x$ of $U(R)$ defined by $b-1=x \pi^{f}$. The definition of $\theta$ and the equality $(\beta-1)^{p}=(b-1)+u v \pi^{e}(\beta-1)$ yield the equality $\theta^{p}=x \pi^{t}$ $+u v^{e-q p+q} \theta$ by an easy computation. Observe that $e-q p+q \geq 1$. For, $e$ $-q p+q \geq 1$ if and only if $q<e / p-1$, which holds if and only if $q p<e p /$ $p-1$; therefore the inequalities $q p<f<e p / p-1$ imply that $e-q p+q \geq 1$. The above expression for $\theta^{p}$ now shows that $\theta$ satisfies a monic polynomial with coefficients in $S$, from which it follows that $\theta$ is itself in $S$. Observe moreover that $\theta$ is a non-unit of $S$ because $t \geq 1$ and $e-q p+q \geq 1$. In order to show that $\theta^{p}$ is in $\pi^{t} U(S)$, it sufficies to show that $e-q p+q \geq t$ since $\theta$ is a non-unit of $S$. Now $e-q p+q \geq t$ if and only if $f \leq e+q$ if and only if $f p \leq e p+f-t$ if and only if $f \leq(e p / p-1)-t / p-1$. Since $0<t / p-1 \leq 1$, we now have that $t \leq e-q p+q$ if and only if $f \leq(e p / p-$ $1)-1$ if and only if $f<e p / p-1$. The assumption that $(f, p)=1$ guarantees that $f<e p / p-1$, and so we may conclude at last that $t \leq e-q p+q$. The equality $\theta^{p}=x \pi^{t}+u v \pi^{e-q p+q} \theta$, together with the inequality $e-q p+q \geq$ $t$ and the fact that $\theta$ is a non-unit, now implies that $\theta^{p}$ is in $\pi^{t} U(S)$ because $x$ is in $U(R)$. Now we may show that $K \supset k$ is wild of degree $p$ by showing that $K \supset k$ has ramification index $p$. Since we have assumed that $f$ and $p$ are relatively prime, we may consider integers $m$ and $n$ such that $m p+n t=1$. An easy computation shows that the element $\Pi$ of $K$ defined by $\Pi=\theta^{n} \pi^{m}$ has the property that $\Pi^{p}$ is in $\pi U(S)$, from which it follows 
that $\Pi$ is an element of $S$, that $K \supset k$ has ramification index $p$, and that $K \supset k$ is wild of degree $p$.

The next step is to show that if $f$ is divisible by $p$ and less than $e p /$ $p-1$, then $K \supset k$ is fiercely ramified of degree $p$. Consider the element $\theta$ of $K$ defined by $\theta=(\beta-1) / \pi^{q}$ where $q=f / p$, and observe that $1 \leq q<e / p-$ 1. In order to prove the assertion we shall show that $\theta$ is an element of $S$ with the property that $\bar{R}(\bar{\theta}) \supset \bar{R}$ is purely inseparable of degree $p$. Let $x$ denote the element of $U(R)$ defined by $b-1=x \pi^{f}$ and observe that $\bar{x}=\bar{x}_{f}$. The equality $(\beta-1)^{p}=(b-1)+u v \pi^{e}(\beta-1)$ (see the beginning of the proof) together with the definition of $\theta$ implies that $\theta^{p}=x+u v \pi^{e-q p+q} \theta$, where $e-p q+q>0$ because $q<e / p-1$; therefore $\theta$ is in $S$ because it satisfies $\mathrm{a}^{\cdot}$ monic polynomial equation with coefficients in $S$. Since $b$ is in normal form by assumption, the fact that $p$ divides $f$ implies that $X^{p}-\bar{x}_{f}$ is irreducible over $\bar{R}_{0}=\bar{R}$. Therefore $\bar{R}(\bar{\theta}) \supset \bar{R}$ is purely inseparable of degree $p$ because $\bar{\theta}^{p}=\bar{x}=\bar{x}_{f}$. We may now conclude that $\bar{S}=\bar{R}(\bar{\theta})$ and that $K \supset k$ is fierce of degree $p$. (Moreover, $S=R[\theta]$ according to part iii) of Lem. 2.4 of [5].)

We show finally that if $f=e p / p-1$ then $K \supset k$ is unramified and we establish necessary and sufficient conditions for $K \supset k$ to have degree $p$. Consider the element $\theta$ defined by $\theta=(\beta-1) / \pi^{e / p-1}$ and observe that $K=k(\theta)$. We shall show that $\theta$ is in $S$, and that $\bar{S}=\bar{R}(\bar{\theta})$ with $\bar{\theta}$ separable over $\bar{R}$. For convenience of notation let $x=x_{f}=x_{e p / p-1}$. Then the definition of $\theta$ together with the equality $(\beta-1)^{p}=(b-1)+u v \pi^{e}(\beta-1)$ (see the beginning of the proof) implies that $\theta^{p}=x+u v \theta$. It follows from the definition of $\theta$ together with the fact that $u$ is in the $R$-module $R\left(1, \beta, \cdots, \beta^{p-2}\right)$ that $u$ is in $R\left(1, \theta, \cdots, \theta^{p-2}\right)$; therefore, the equality $\theta^{p}-u v \theta-x=0$ gives rise to a monic polynomial $f(X)$ in $R[X]$ having $\theta$ as a root, from which it follows that $\theta$ is in $S$. Observe that $\bar{f}(X)=X^{p}+\bar{v} X-\bar{x}$ in $\bar{R}[X]$ because $\bar{u}=-\overline{1}$, and that $\bar{f}(X)$ is a separable polynomial because $\bar{f}^{\prime}(X)=\bar{v} \neq \overline{0}$. We proceed to show that $[K: k]=p$ if and only if $\bar{f}(X)$ is irreducible over $\bar{R}=\bar{R}_{0}$, and that $K=k$ otherwise. If $\bar{f}(X)$ is reducible over $\bar{R}$, then $f(X)$ is reducible over $R$ by Hensel's lemma because $R$ is complete and $\bar{f}(X)$ is separable; the reducibility of $f(X)$ over $R$ implies that $\operatorname{deg}_{k} \theta<p$ from which it follows that $\beta$ is in $k$ and that $K=k$. If, on the other hand, $\bar{f}(X)$ is irreducible over $\bar{R}$, then the separability of $\bar{f}(X)$ implies that $K \supset k$ is unramified of degree $p$ (with $\bar{S}=\bar{R}(\bar{\theta})$ and $S=R[\theta]$ ) according to Prop. 1 p. 25 of [2]. 
The above observations combine to establish the truthfulness of the proposition.

Observe that the equation $X^{p}+\bar{\imath} X-\bar{x}=\overline{0}$ of Prop. 1.7 is essentially an Artin-Schreier equation (see p. 80 of [4]). For, consider the elements $v, v_{1}$, and $v_{0}$ defined by $v \pi^{e}=p, \quad v_{1} \pi^{e / p-1}=\zeta-1$, and $v_{0}(\zeta-1)^{p-1}=p$ where $\zeta$ denotes as usual a primitive $p^{t h}$ root of unity. An easy computation shows that $v=v_{1}^{p-1} v_{0}$, so that $\bar{v}=-\bar{v}_{1}^{p-1}$ because $\bar{v}_{0}=-\overline{1}$ (see p. 158 of [1]). The change of variable $Y=X / \bar{v}_{1}$ yields the Artin-Schreier equation $Y^{p}-Y-\bar{x} /$ $\bar{v}_{1}^{p}=\overline{0}$.

The following expressions for the integral closure $S$ of $R$ in $K$ follow at once from the proof of Prop. 1.7.

Remark 1.8. Let $b=1+\sum x_{i} \pi^{i}$ denote an element of $U^{(f)}-U^{(f+1)}(1 \leq$ $f \leq e p / p-1)$ in normal form. Consider the unique integers $q$ and $t$ for which $f=q p+t$ with $0 \leq t<p$, and define $\theta=(\beta-1) / \pi^{q}$.

i) If $f$ is relatively prime to $p$, then $S=R[\Pi]$ where $\Pi=\theta^{n} \pi^{m}$ for integers $m$ and $n$ satisfying $m p+n t=1$.

ii) If $p$ divides $f$, then $S=R[\theta]$.

Lemma 1.9. Consider elements $b_{1}$ and $b_{2}$ of $U^{(1)}$ in normal form, where $b_{1}$ is in $U^{\left(f_{1}\right)}-U^{\left(f_{1}+1\right)}$ and $b_{2}$ is in $U^{\left(f_{2}\right)}-U^{\left(f_{2}+1\right)}$. If $k\left(b_{1}{ }^{1 / p}\right)=k\left(b_{2}{ }^{1 / p}\right)$, then $f_{1}=f_{2}$.

Proof. Since $k\left(b_{1}{ }^{1 / p}\right)=k\left(b_{2}{ }^{1 / p}\right)$ by hypothesis, an application of Prop. 1.7 shows that $f_{1}$ and $f_{2}$ are both relatively prime to $p$, are both divisible by $p$ and less than $e p / p-1$, or are both equal to $e p / p-1$.

Consider an equality $k\left(b_{1}{ }^{1 / p}\right)=k\left(b_{2}{ }^{1 / p}\right)$ with $f_{1}$ and $f_{2}$ relatively prime to $p$. We shall show that $f_{1}=f_{2}$ by contradiction. Assume that $f_{1}<f_{2}$. Since $k\left(b_{1}{ }^{1 / p}\right)=k\left(b_{2}{ }^{1 / p}\right)$, we may consider an element $c$ of $k$ such that $b_{1}=$ $c^{p} b_{2}{ }^{n}$ for some integer $n$ relatively prime to $p$, (see Lem. 3 p. 90 of [2]). Observe that $c^{p}$ is in $U^{\left(f_{1}\right)}-U^{\left(f_{1}+1\right)}$ because $b_{1}$ is in $U^{\left(f_{1}\right)}-U^{\left(f_{1}+1\right)}, b_{2}^{n}$ is in $U^{\left(f_{2}\right)}$, and $f_{1}<f_{2}$, so that $k(c) \supset k$ is wild of degree $p$ according to Prop. 1.7. This contradiction shows that $f_{1}=f_{2}$.

Now consider an equality $k\left(b_{1}{ }^{1 / p}\right)=k\left(b_{2}{ }^{1 / p}\right)$ with $f_{1}$ and $f_{2}$ divisible by $p$ and less than $e p / p-1$, and assume that $f_{1}<f_{2}$. Once again we consider an element $c$ in $k$ such that $b_{1}=c^{p} b_{2}{ }^{n}$ for some integer $n$ relatively prime to $p$. Since $b_{2}^{n}$ is in $U^{\left(f_{2}\right)}$, we have that $c^{p} \equiv b_{1} \bmod \pi^{f_{2}} R$ from which it follows that $c^{p}$ is of the form $c^{p}=1+y \pi^{f_{1}}$ with $\bar{y}=\bar{x}_{f_{1}}$ because $f_{1}<f_{2}$. 
The irreducibility of $X^{p}-\bar{y}$ over $\bar{R}$ now implies that $k(c) \supset k$ is fierce of degree $p$ by Prop. 1.7. This contradiction shows that $f_{1}=f_{2}$, and this completes the proof.

The following proposition follows at once from the four lemmas established above.

Proposition 1.10. Let $k$ denote the quotient field of a complete discrete rank one valuation ring $R$ containing a primitive $p^{\text {th }}$ root of unity, where $p=\operatorname{char} \bar{R}$, and consider a Galois extension $K \supset k$ of degree $p$. Then there exists a unique integer $f$ $(-1 \leq f \leq e p / p-1)$ such that $K \supset k$ is one of the following forms:

i) $K=k\left(b^{1 / p}\right)$ for some element $b$ of $U^{(f)}$ with $f=-1$

ii) $K=k\left(b^{1 / p}\right)$ for some element $b$ of $U^{(f)}$ with $f=0$ for which $X^{p}-\bar{b}$ is irreducible over $\bar{R}$

iii) $K=k\left(b^{1 / p}\right)$ for some element $b$ of $U^{(f)}-U^{(f+1)}$ in normal form, (where $1 \leq$ $f \leq e p / p-1)$.

Definition. The unique integer $f$ satisfying $-1 \leq f \leq e p / p-1$ defined for each Galois extension $K \supset k$ of degree $p$ by Prop. 1.10 is called the absolute field exponent of $K \supset k$ and is denoted by $f(K / k)$.

The following theorem has now been established.

Theorem 1.11. Let $f=f(K / k)$ denote the absolute field exponent of a Galois extension $K \supset k$ of degree $p$. Then

i) $K \supset k$ is wildly ramified if and only if $f$ is relatively prime to $p$

ii) $K \supset k$ is fiercely ramified if and only if $p$ divides $f$ and $f<e p / p-1$

iii) $K \supset k$ is unramified if and only if $f=e p / p-1$.

We terminate this section with some observations concerning the relationship between the field exponent $x=x(K / k)$ (see Section 1 of [5]) and the absolute field exponent $f=f(K / k)$ of a Galois extension $K \supset k$ of degree $p$. These observations follow at once from the definitions of $x$ and $f$. Recall that $-1 \leq x \leq p$ and that $-1 \leq f \leq e p / p-1$.

Remark 1.12. Let $x$ denote the field exponent and $f$ the absolute field exponent of a Galois extension $K \supset k$ of degree $p$.

i) If $e=p-1$, then $x=f$.

ii) If $-1 \leq f \leq p$, then $x=f$. 
iii) If $-1 \leq x \leq p-1$, then $x=f$.

2. The ramification number, the absolute field exponent, and the conductor number. As usual, $K \supset k$ denotes a Galois extension of degree $p$ where $k$ is the quotient field of a complete discrete rank one valuation ring $R$ which contains a primitive $p^{t h}$ root of unity and whose residue class field has characteristic $p$. In Section 2 of [5], the author has assigned to each such extension $K \supset k$ an integer $g$ with $0 \leq g \leq(e / p-1)-1$ called the conductor number of $K \supset k$. Prop. 3.1 of [5] presents expressions for the ramification number $i$ of $K \supset k$ in terms of its conductor number $g$.

The purpose of this section is to determine the relationships between the absolute field exponent of an extension and its ramification and conductor numbers.

Proposition 2.1. Let $f$ denote the absolute field exponent of a Galois extension $K \supset k$ of degree $p$, and let $i$ denote the ramification number of $K \supset k$.

i) If $f=-1$, then $i=e p / p-1$.

ii) If $p$ divides $f$, then $i=(e / p-1)-f / p-1$.

iii) If $f>0$ and $(f, p)=1$, then $i=(e p / p-1)-f$.

Proof. Let $x=x(K / k)$ denote the field exponent of $K \supset k$ (see Section 1 of [5]). If $f=-1$, then $x=-1$ (see Remark 1.12). According to part ii) of Prop. 3.1 of [5], $i=e p / p-1$ when $x=-1$, and this proves statement $\mathrm{i}$ ). (Or, the reader may refer to Exer. 4 p. 79 of [4]).

To prove statement ii) we first consider the case when $p$ divides $f$ and $f<e p / p-1$; in this case $K \supset k$ is fierce and the integral closure $S$ of $R$ in $K$ is given by $S=R[\theta]$ where $\theta=(\beta-1) / \pi^{q}$ and $q=f / p$ (see Prop. 1.7 and Remark 1.8). Consider some primitive $p^{t h}$ root of unity $\zeta$ and let $\sigma$ denote the element of the Galois group $G(K / k)$ defined by $\sigma(\beta)=\zeta \beta$; observe that $\sigma$ is in the $i^{t h}$ ramification group $G_{i}$ of $K \supset k$ if and only if $\sigma(\theta) \equiv \theta \bmod \pi^{i+1} S$. An easy computation shows that $\sigma\left((\beta-1) / \pi^{q}\right) \equiv(\beta-1) / \pi^{q} \bmod \pi^{i+1} S$ if and only if the element $\zeta-1$ is in $\pi^{i+q+1} S$, which in turn holds if and only if $\pi^{e / p-1}$ is in $\pi^{i+q+1} S$ because $\zeta-1$ is in $\pi^{e / p-1} U(S)$. The fact that $\pi^{e / p-1}$ is in $\pi^{i+q+1} S$ if and only if $i \leq(e / p-1)-q-1$ shows that $\sigma$ is in $G_{i}$ if and only if $i \leq(e / p-1)-q-1$.

In the case when $f=e p / p-1$, the extension $K \supset k$ is unramified according to Prop. 1.7. It is well known that the ramification number of an 
unramified extension is -1 . The observation that $(e / p-1)-f / p-1=-1$ when $f=e p / p-1$ completes the proof of statement ii).

In the case when $f>0$ and $(f, p)=1$, the extension $K \supset k$ is wild according to Prop. 1.7. Let $\theta=(\beta-1) / \pi^{q}$ where $q$ is defined by $f=q p+t$ with $0 \leq t<p$. Recall (Remark 1.8) that the integral closure $S$ of $R$ in $K$ is given by $S=R[\Pi]$ where $\Pi=\theta^{n} \pi^{m}$ for integers $m$ and $n$ satisfying $m p+$ $n t=1$. Once again let $\zeta$ denote a primitive $p^{t h}$ root of unity and $\sigma$ the element of $G(K / k)$ for which $\sigma(\beta)=\zeta \beta$. Observe that $\sigma$ is in the $i^{t h}$ ramification group $G_{i}$ of $K \supset k$ if and only if $\sigma(\Pi) / \Pi \equiv 1 \bmod \Pi^{i} S$. By substituting $\left((\beta-1) / \pi^{q}\right)^{n} \pi^{m}$ for $\Pi$ one can obtain the equality $\sigma(\Pi) / \Pi=(\sigma(\beta-1) /(\beta-1))^{n}$, so that $\sigma$ is in $G_{i}$ if and only if $(\sigma(\beta-1) /(\beta-1))^{n} \equiv 1 \bmod \Pi^{i} S$. We proceed to show that $(\sigma(\beta-1) /(\beta-1))^{n}-1$ is in $\Pi^{(e p / p-1)-f} U(S)$. First observe that $\beta-1$ is in $\Pi^{f} U(S)$. For, $\theta$ is in $\Pi^{t} U(S)$ because $\theta^{p}$ is in $\pi^{t} U(S)$, (see paragraph two of the proof of Prop. 1.7), and so the definition $\beta-1=\theta \pi^{q}$ implies that $\beta-1$ is in $\prod^{q p+t} U(S)=\prod^{f} U(S)$. Since $\sigma(\beta-1)-(\beta-1)$ is in $\prod^{e p / p-1} U(S)$ and $\beta-1$ is in $\prod^{f} U(S)$, we have that $\sigma(\beta-1) /(\beta-1)-1$ is in $\Pi^{(e p / p-1)-f} U(S)$. Therefore $(\sigma(\beta-1) /(\beta-1))^{n}-1$ is in $\Pi^{(e p / p-1)-f} U(S)$ because $n$ is relatively prime to $p$. The above observations combine to give us that $\sigma$ is in $G_{i}$ if and only if $\Pi^{(e p / p-1)-f}$ is in $\Pi^{i} S$, i.e. $\sigma$ is in $G_{i}$ if and only if $i \leq(e p / p-1)-f$, and this completes the proof of part iii).

It remains to study the relationship between the absolute field exponent $f$ and the conductor number $g$. For this the following definition is useful.

Definition. Let $f$ denote the absolute field exponent of a Galois extension $K \supset k$ of degree $p$. If $f \geq 0$, then the quotient number $q$ and the $r$ mainder number $t$ of $K \supset k$ are the unique integers $q$ and $t$ such that $f=q p$ $+t$ with $0 \leq t<p$; if $f=-1$, we define $q=0$ and $t=1$.

Proposition 2.2. Let $q$ denote quotient number and $g$ the conductor number of $a$ Galois extension $K \supset k$ of degree $p$. If $K \supset k$ is unramified then $q=g+1$. Otherwise, $q=g$.

Proof. If $K \supset k$ is unramified, then $f=e p / p-1$ (Thm. 1.11) and so $q=e / p-1$. On the other hand, $g=(e / p-1)-1$ (Cor. 2.7 of [5]); therefore $q=e / p-1=g+1$ in the unramified case.

We shall make use of Prop. 2.1 to prove that $q=g$ when $K \supset k$ is fiercely ramified or wildly ramified.

If $K \supset k$ is fierce, then the ramification number $i$ of $K \supset k$ is given on 
the one hand by $i=(e / p-1)-q-1$ (Prop. 2.1), and on the other hand by $i=(e / p-1)-g-1$ (Prop. 3.1 of [5]), from which it follows that $q=g$.

Now let $x$ denote the field exponent of a wildly ramified extension $K \supset k$. If $f=-1$, then $q=0=g$. For, $x=-1$ when $f=-1$ (Remark 1.12) so that $g=0$ (see p. 155 of [5]); and $q=0$ when $f=-1$ according to the above definition of $q$. If $f \neq-1$, then $i=(e p / p-1)-f$ by Prop. 2.1; and, the fact that $x \neq-1$ when $f \neq-1$ (Remark 1.12) implies that $i=(e p / p-1)$ $-g p-h$ where $1 \leq h \leq p-1$ (Prop. 3.1 of [5]). The equalities $i=(e p / p-$ 1) $-q p-t$ and $i=(e p / p-1)-g p-h$ together with the inequalities $1 \leq h, t$ $\leq p-1$ imply that $q=g$ (and $h=t)$.

\section{REFERENCES}

[1] E. Artin and J. Tate, Class field theory, Benjamin, (1967).

[2] J.W.S. Cassels and A. Frolich, Algebraic Number Theory, Thompson, (1967).

[3] M. Nagata, Local Rings, Wiley, (1962).

[4] J.-P. Serre, Corps Locaux, Paris, Hermann, (1962).

[ 5 ] S. Williamson, Ramification theory for extensions of degree p, Nagoya Math. J. Vol. 41 (1971), pp. 149-168.

Regis College

Weston, Massachusetts 